\title{
Poster Abstracts (Session 5)
}

\section{(Full Posters are available at http://www.journals.cambridge.org/jid_IAU)}

\section{Absolute Parameters of the O-type Eclipsing Binary V1007 Sco}

\author{
S. Nesslinger ${ }^{1}$, H. Drechsel ${ }^{1}$, R. Lorenz ${ }^{1}$, P. Harmanec ${ }^{3}$, P. Mayer ${ }^{2}$, and M. Wolf ${ }^{2}$ \\ ${ }^{1}$ Dr. Remeis Observatory, University of Erlangen-Nuremberg, Bamberg, Germany, \\ ${ }^{2}$ Astronomical Institute of the Charles University, Prague, Czech Republic, \\ ${ }^{3}$ Astronomical Institute, Academy of Sciences, Ondrejov, Czech Republic
}

A definitive set of orbital and physical parameters of the binary system V1007 Sco is presented. Both stars are classified as O7 giants. Light and RV curves were simultaneously analyzed with PHOEBE, a modern WD-based solution code. A statistical approach yielded realistic error estimates. Orbital eccentricity is 0.12 with an apsidal motion period of 112 years.

\section{Draconis: Masses and Radii of Very Low Mass Stars}

\author{
J.C. Morales ${ }^{1,3}$, I. Ribas ${ }^{1,2}$, C. Jordi ${ }^{1,3}$, G. Torres ${ }^{4}$, E.F. Guinan ${ }^{5}$, and D. Charbonneau ${ }^{4}$ \\ ${ }^{1}$ Institut d'Estudis Espacials de Catalunya, Barcelona, Spain \\ ${ }^{2}$ Institut de Ciències de l'Espai - CSIC, Bellaterra, Spain, \\ ${ }^{3}$ Dept. d'Astronomia i Meteorologia, Universitat de Barcelona, Barcelona, Spain, \\ ${ }^{4}$ Harvard-Smithsonian Center for Astrophysics, Cambridge, MA, United States, \\ ${ }^{5}$ Dept. of Astronomy, Villanova University, Villanova, PA, United States
}

Eclipsing binaries provide the only high-accuracy $(\sim 1-2 \%)$ measures of stellar masses and radii, making them suitable for relevant tests of the models of structure and evolution of stars. At the low-mass end of the main sequence, current results have shown that models predict radii smaller by $\sim 10 \%$ and effective temperatures larger by $\sim 5 \%$ than observed. Although no definitive explanation of such differences has been yet reached, some evidence suggests that the disagreement could be related to the strong activity and intense magnetic fields in these stars. In this work we study CM Draconis, one of the least massive eclipsing binaries known. Their components are very similar, both with dM4 spectral type, and masses and radii of about $0.23 \mathrm{M}_{\odot}$ and $0.25 \mathrm{R}_{\odot}$, respectively. We have analyzed light curves in the $R$ and $I$ bands taken between 1975 and 2004 with a total of 25051 measurements. To calculate the fundamental properties of this system with accuracies better than $1 \%$ we have considered spots on the surface of the stars and modeled the light curve variations accordingly. From the resulting accurate masses and radii we plan to carry out a thorough test of the models for these fully convective stars. This will be especially interesting since the mechanism driving magnetic activity is thought to be different from that of more massive stars. In addition, the extended time-span of the observations has led to the detection of apsidal motion in this system, which has a slightly eccentric orbit. This provides a further check on models through the determination of the internal structure of the stars 


\title{
A Mass Estimate in a Sample of Double Stars
}

S. Ninkovic and Z. Cvetkovic

Astronomical Observatory, Belgrade, Yugoslavia (Serbia and Montenegro)

The total masses of binaries are calculated on the basis of their orbital elements from the Sixth Catalog of Orbits of Visual Binary Stars. They are then compared with the values resulted from the mass-luminosity relation for the Main Sequence where as the input data are used: trigonometric parallax, total apparent magnitude of the pair (source Hipparcos Catalog) and magnitude difference (sources Hipparcos Catalog and Photometric Magnitude Difference Catalog). It seems that for the pairs indicated as having qualitative orbital elements the agreement between the total-mass values obtained in these two ways is satisfactory.

\section{GQ Lup, 2M1207, and AB Pic: Planet Companion Candidates Imaged Directly and their Relevance in Orbital Dynamics and Mass Estimation via Theoretical Models}

\author{
R. Neuhaeuser ${ }^{1}$, M. Mugrauer ${ }^{1}$, and A. Seifahrt ${ }^{2}$ \\ ${ }^{1}$ AIU University Jena, Jena, Germany, \\ ${ }^{2}$ ESO Garching, Garching, Germany
}

In 2005, evidence was presented for three exo-planets imaged directly: GQ Lup, 2M 1207, and AB Pic. In all three cases, a faint red object is co-moving with a young nearby star. The masses of these companions are determined through theoretical models, which are under dispute and have not yet been tested successfully in the relevant parameter range of young ages and low masses. We show that being co-moving with another star and having a late spectral type is necessary, but not sufficient for being gravitationally bound. We discuss the relevance of these three wide visual binary systems for orbital dynamics and testing theoretical models. We will also present new images from 2005 and 2006 to investigate orbital motion of GQ Lup b around GQ Lup A.

\section{Low-mass Eclipsing Binaries to Refine Barnes-Evans-like Relations}

\section{P.J. Amado}

Univ. Granada-IAA(CSIC), Granada, Spain

The relation of the surface brightness, a parameter related to the apparent magnitude and the angular diameter of a star, with a colour index was first calibrated by Wesselink (1969) and later refined by Barnes \& Evans (1976). This calibration has a very wide range of applicability amongst which is the evaluation of various stellar parameters. In this work, we use a number of low-mass eclipsing binaries whose parameters have been accurately determined to refine the Barnes-Evans-like relation $F_{V}-I_{c}-K$ ) introduced by Amado et al. (1999), a relation more suitable for the low temperature of cool stars. 


\title{
Eclipsing Binaries as a Test for Synthetic Photometry
}

\author{
U. Heiter ${ }^{1}$, B. Smalley ${ }^{2}$, Ch. Stütz ${ }^{3}$, F. Kupka ${ }^{4}$, and O. Kochukhov ${ }^{1}$ \\ ${ }^{1}$ Department of Astronomy and Space Physics, Uppsala University, Uppsala, Sweden, \\ ${ }^{2}$ Astrophysics Group, School of Chemistry and Physics, Keele University, Keele, \\ United Kingdom, \\ ${ }^{3}$ Institute for Astronomy, University of Vienna, Vienna, Austria, \\ ${ }^{4}$ Max-Planck-Institute for Astrophysics, Garching, Germany
}

Narrow-band photometry is a viable tool to characterize large numbers of stars. The connection between observed colors and astrophysical parameters has to rely on synthetic photometry calculated from stellar atmosphere models. Here, we present synthetic $\mathrm{H} \beta$ indices calculated from 1D model atmospheres, which implement various treatments of convection. The calculated indices are transformed to the standard system using observed medium-resolution spectra from recently published stellar libraries. We test how well the synthetic photometry reproduces observed indices by using a number of eclipsing binary systems. For these stars, atmospheric parameters can be determined independently from the models with highest possible accuracy. As a preliminary conclusion, the computed indices deviate from the observed ones by an amount expected from the observational errors and the accuracy of the atmospheric parameters.

Note: The full text of this paper will be published in the proceedings of IAU Symposium 239, Convection in Astrophysics.

\section{Looking for Exoplanets in Bright Stars with Small Field-of-View Detectors}

Eder Martioli and Francisco Jablonski

Instituo Nacional de Pesquisas Espaciais - Divisâo de Astrofisica, Sâ José dos Campos, $S P$, Brasil

Differential photometry is a robust technique for ground-based observations of transits since it sorts out slow variations of sky transparency as well as other first order effects that are common to all stars in the field-of-view (FOV) of the imaging detector. To work properly, differential photometry has to obey a few requirements like similar brightness of the target and reference stars, similar colors and a relative proximity in the plane of the sky to avoid sensitivity variations like those caused by vignetting. It happens that for bright stars these conditions are hardly met. Typical CCDs in a $\sim 60$-cm class telescope give a FOV of $\sim 10$ arcmin and this is not enough to have suitable reference stars in the same image frame. Also, bright $(V<7)$ stars tend to saturate the detector for the shortest practical integration times. To minimize these problems, we tested an instrumental setup in which half of the detector is covered with a neutral density $(D=2.3)$ filter. We report CCD observations on which we achieved mmag precision for bright systems that are not known to show transits, like $\tau$ Boo, 55 Cnc and HD 162020, as well as the known case of HD 209458. 


\title{
Multiplicity Study of Exoplanet Host Stars: The HD 3651 AB System
}

\author{
M. Mugrauer ${ }^{1}$, A. Seifahrt ${ }^{2}$, R. Neuhaeuser ${ }^{1}$, T. Mazeh $^{3}$, and T. Schmidt ${ }^{1}$ \\ ${ }^{1}$ Astrophysikalisches Institut, Universität Jena, Jena, Germany, \\ ${ }^{3}$ European Southern Observatory, Garching, Germany, \\ ${ }^{3}$ Tel Aviv University, Tel Aviv, Israel
}

We present new results from our ongoing multiplicity study of exoplanet host stars. We found new stellar companions of the exoplanet host stars GJ 3021 and HD 40979 and present our imaging and spectroscopic data of the wide companion of the exoplanet host star HD 27442. GJ 3021 is a new close planet hosting binary system with a M3-M5 stellar companion $\left(\sim 0.125 \mathrm{M}_{\odot}\right)$ which is separated from its primary by only $70 \mathrm{AU}$. In contrast, HD 40979 is one of the widest planet hosting stellar systems known today with a projected separation of $\sim 6400 \mathrm{AU}$. We present our observations of the wide companion HD 40979B, which turned out to be a stellar pair composed of a $\sim 0.8 \mathrm{M}_{\odot}$ and $\mathrm{a} \sim 0.4 \mathrm{M}_{\odot}$ dwarf with a projected separation of $\sim 130$ AU. Hence, HD 40979 is a new member of the small group of planet hosting triple star systems known today. Finally, we present our observations of the planet hosting subgiant HD 27442, which has a co-moving companion with a projected separation of $\sim 240 \mathrm{AU}$. The $V$ - and $H$-band magnitudes of this faint companion are fully consistent with a relatively young, hot white dwarf, with an effective temperature of $\sim 14400 \mathrm{~K}$, and cooling age of $\sim 220 \mathrm{Myr}$. With follow-up spectroscopy which shows Hydrogen absorption features in its optical and infrared spectra, we confirm the white dwarf nature of this companion. With the subgiant exoplanet host star and its white-dwarf companion, HD 27442AB is the most evolved planet hosting stellar system presently known.

\section{Preliminary Orbit and Masses of the Nearby Binary L Dwarf GJ 1001BC}

D.A. Golimowski ${ }^{1}$, D. Minniti ${ }^{2}$, T.J. Henry ${ }^{3}$, and H.C. Ford ${ }^{1}$

${ }^{1}$ Johns Hopkins University, Baltimore, MD, United States,

${ }^{2}$ Pontificia Universidad Catolica de Chile, Santiago, Chile,

${ }^{3}$ Georgia State University, Atlanta, GA, United States

We present preliminary results of a continuing VLT program to map the orbit of the nearby binary L4.5 dwarf GJ 1001BC (LHS 102BC). Since discovering its duplicity in 2002 and 2003 using HST's NICMOS and ACS, we have obtained high-resolution images of GJ 1001BC at three epochs between October 2004 and November 2005, using the NAOS/CONICA system at VLT-UT4 (Yepun). Our HST and VLT images cover 75\% of GJ 1001BC's 4-year orbit. A least-squares fit of a Keplerian orbit yields a combined binary mass of $0.100 \pm 0.026 \mathrm{M}_{\odot}$ for a tentative parallactic distance measurement of $13.0 \pm 0.7 \mathrm{pc}$ to the M dwarf GJ 1001A. Hypothetically assuming a 3:2 mass ratio for the nearly equal-luminosity $\mathrm{L}$ dwarfs, we estimate masses of $0.060 \pm 0.016 \mathrm{M}_{\odot}$ and 0.040 $\pm 0.010 \mathrm{M}_{\odot}$ for GJ 1001B and C, respectively. If these preliminary values are sustained by our continuing orbit and parallax measurements, then GJ $1001 \mathrm{C}$ will be the least massive L dwarf for which a dynamical mass has been measured. 


\title{
Near-Infrared Light Curves of a Young, Eclipsing Binary of Brown Dwarfs
}

\author{
Y. Gómez Maqueo Chew ${ }^{1}$, K.G. Stassun ${ }^{1}$, R. Mathieu ${ }^{2}$, and L.P. Vaz ${ }^{3}$, \\ ${ }^{1}$ Vanderbilt University, Nashville, TN, United States, \\ ${ }^{2}$ University of Wisconsin, Madison, WI, United States, \\ ${ }^{3}$ Universidade Federal de Minas Gerais, Belo Horizonte, Brazil
}

We present the near-infrared light curves for an eclipsing binary system,

2MASS J05352184-0546085, in which both components are brown dwarfs (Stassun et al. 2006). The system is a member of the Orion Nebula Cluster, and therefore has a likely age of only a few million years.

We model light curves using a Wilson-Devinney based code to derive fundamental system properties, including effective temperatures and radii. Our analysis includes $J H K$ light curves obtained with the 1.3-m SMARTS telescope at CTIO in Chile.

A thorough spectroscopic and photometric analysis of eclipsing binary systems yields highly accurate properties of the system and of its components, in such a manner that they are determined independently of their distance and other assumptions. The masses of both components, 0.054 and $0.034 \mathrm{M}_{\odot}$, have been measured with accuracies better than $10 \%$.

This system is of particular interest because it provides with the measurements of the two least massive of pre-main-sequence objects known to date, and consequently provides useful data for testing the predictions of current early evolution and star formation models.

\section{RZ Cassiopeia: an Eclipsing Binary with a Pulsating Component}

\author{
Alex Golovin ${ }^{1}$ and Elena Pavlenko ${ }^{2}$ \\ ${ }^{1}$ Kiyv National Taras Shevchenko University; visiting astronomer of the \\ Crimean Astrophysical Observatory, Kyiv, Ukraine, \\ ${ }^{2}$ Crimean Astrophysical Observatory, Nauchny, Ukraine
}

We report time-resolved $V R$-band CCD photometry of the eclipsing binary RZ Cas obtained with a 38-cm Cassegrain telescope at the Crimean Astrophysical Observatory during July 2004 - October 2005.

Obtained lightcurves clearly demonstrates rapid pulsations with a period about 22 minutes. Periodogram analysis of such oscillations also is reported. On the 12, January, 2005 we observed rapid variability with higher amplitude $\left(\sim 0^{\mathrm{m}} \cdot 1\right)$ that, perhaps, may be interpreted as high-mass-transfer-rate event and inhomogeneity of accretion stream.

Follow-up observations (both, photometric and spectroscopic) of RZ Cas are strictly desirable for more detailed study of such event. 


\title{
Orbital Period Changes of OB-type Contact Binaries and Their Implications for the Triplicity, Formation and Evolution of This Type of Binary Star
}

\author{
S.-B. Qian ${ }^{1}$, J.M. Kreiner ${ }^{2}$, L. Liu ${ }^{1}$, J.-J. He ${ }^{1}$, L.-Y. Zhu ${ }^{1}$, J.-Z. Yuan ${ }^{1}$, and Z.-B. Dai ${ }^{1}$ \\ ${ }^{1}$ Yunnan Observatory, Kunming, China, \\ ${ }^{2}$ Cracow Pedagogical University, Cracow, Poland
}

Orbital period variations of 9 well-observed OB-type contact binary stars, LY Aur, BH Cen, V382 Cyg, V729 Cyg, AW Lac, TU Mus, RZ Pyx, V701 Sco and CT Tau, are investigated in detail. Of the nine systems, V701 Sco and CT Tau are two contact binaries containing twin components with a mass ratio of unity, LY Aur and V729 Cyg have the longest period among contact binary stars $(\mathrm{P}=4.0$ and 6.6 days, respectively), and BH Cen and V701 Sco are members of the two extremely young galactic clusters IC 2994 and NGC 6383. It was discovered that, apart from the two systems with twin components (V701 Sco and CT Tau), the orbital periods of the remaining 7 binary stars show a long-term increase. This is different from the situations of the late-type (W UMa-type) contact binaries where both secular period increase and decrease are usually encountered, indicating that magnetic fields may play an important role in causing the long-term period decrease of W UMa-type contact binary stars. The fact that no longterm continuous period variations were found for V701 Sco and CT Tau may suggest that contact binaries with twin components can be in an equilibrium. Based on the rates of period changes $(\mathrm{dP} / \mathrm{dt})$ of the 7 sample binary stars, statistical relations between $\mathrm{dP} / \mathrm{dt}$ and orbital period $(\mathrm{P})$ and the mean density of the secondary component were found. Our results suggest that the period increases of the short-period systems $(\mathrm{P}<2$ days $)$ may be mainly caused by a mass transfer from the less massive component to the more massive one, while for the long-period ones ( $\mathrm{P}>2$ days), LY Aur and V729 Cyg, their period increases may be resulted from a combination of stellar wind and mass transfer from the secondary to the primary.

Meanwhile, cyclic period changes are found for all of the nine binary systems. Those periodic variations can be plausibly explained as the results of light-travel time effects suggesting that they are triple systems. The astrophysical parameters of the tertiary components in the nine systems have been determined. The tertiary components in the seven binaries, BH Cen, V382 Cyg, AW Lac, TU Mus, RZ Pyx, V701 Sco and CT Tau, may be invisible, while those in LY Aur and V729 Cyg may be the fainter visual companions in the two systems. It is possible that the tertiary components in those binaries played an important role for the formations and evolutions of the contact configurations by bringing angular momentum out from the central systems. Thus they have short initial periods and can evolve into contact configurations in a short timescale. 


\title{
Quasi-Molecular K-H $\mathrm{H}_{2}$ Absorption as an Alternative to the Resurgence of CaH Bands in the Spectra of T-Type Dwarfs: is the Cloud-Clearing Scheme at Stake?
}

\author{
F. Allard ${ }^{1}$, N.F. Allard ${ }^{2}$, C.M.S. Johnas ${ }^{3}$, P.H. Hauschildt ${ }^{3}$, D. Homeier ${ }^{4}$, J.K. Kielkopf ${ }^{5}$, \\ and F. Spiegelman ${ }^{6}$ \\ ${ }^{1}$ Centre de Recherche Astronomique de Lyon, Lyon, France, \\ ${ }^{2}$ Institut d'Astrophysique de Paris, Paris, France, \\ ${ }^{3}$ Hamburger Strenwarte, Hamburg, Germany, \\ ${ }^{4}$ Institut für Astrophysik Götingen, Götingen, Germany, \\ ${ }^{5}$ University of Louisville, Louisville, KY, United States, \\ ${ }^{6}$ Universite Paul Sabatier, Toulouse, France
}

As brown dwarfs cool off with time, their atmospheres become denser and more transparent, allowing the emitted thermal flux to escape from deeper atmospheric layers. Burgasser et al. (2002) have investigated and classified the red spectra of $\mathrm{T}$ dwarfs in a spectral sequence where a resurgence of the hydride bands, after disappearing in the $\mathrm{M}$ to $\mathrm{L}$ spectral transition, occur between the late $\mathrm{L}$ to $\mathrm{T}$ before disappearing again in the late $\mathrm{T}$ dwarfs. $\mathrm{CaH}$ for example is identified in mid- $\mathrm{T}$ dwarfs at around $0.7 \mu \mathrm{m}$ (Burgassser 2003). The authors explain this resurgence by a cloud-clearing scheme where holes would allow to see the $\mathrm{CaH}$ from deeper enriched layers, while it is settled out from the uppermost atmospheric layers seen on the rest of the brown dwarf surface.

We present the first synthetic spectra of $\mathrm{T}$ dwarfs including a semi-classical modelling of the pressure broadening of alkalis lines ( $\mathrm{Na} \mathrm{I} \mathrm{D,} \mathrm{Li} \mathrm{I,} \mathrm{K} \mathrm{I,} \mathrm{Rb} \mathrm{I,} \mathrm{and} \mathrm{Cs} \mathrm{I} \mathrm{fundamental} \mathrm{res-}$ onance doublets) by molecular hydrogen and helium, the most important species in these atmospheres. We compare the models to the $\mathrm{T}$ dwarfs red optical spectra of Burgasser et al. (2003) and we find that the $0.7 \mu \mathrm{m}$ feature has been wrongly identified to CaH. In particular, the very strong KI resonance transition doublet at $0.77 \mu \mathrm{m}$ explains by itself this absorption feature by producing a quasi-molecular satellite absorption feature at this wavelength. The strength of this satellite is very sensitive to the density of perturbers in the lower photosphere and to the background opacity provided by the Na I D red wing, which explains naturally both its apparition in late $\mathrm{L}$ dwarfs and its vanishing in late $\mathrm{T}$ dwarfs.

We find in conclusion that no cloud-clearing scheme or non-equilibrium processes is necessary to explain this absorption feature, and the evolution of the red optical spectrum of $\mathrm{T}$ dwarfs. And this should teach us caution about these atmospheres often too enthusiastly considered planetary. MHR 3D convection models are nevertheless underway to estimate the likelihood of cloud-clearings in late $\mathrm{L}$ and $\mathrm{T}$ dwarfs. 


\title{
Search for Exoplanets and Variables in the Open Cluster NGC 381
}

\author{
J.-H. Hu and W.-H. Ip
}

Institute of Astronomy, National Central University, Jhongli City, Taiwan

We present the result of a search for exoplanets and variable stars in the open cluster star fields, NGC 381, with the Lulin One-meter Telescope (LOT), Taiwan. The main scientific goal is to use time series CCD photometry measurements to detect exoplanets via transit effects. Observations of open clusters would give us important information on the formation of planets in different stellar environments. The secondary scientific goal is to discover and study variable stars with the similar data analysis process. Several program stellar clusters have been observed. Four variable candidates were discovered in the NGC 381 star field. One of them was identified as one of the suspected cluster member, CT 123, by Crinklaw \& Talbert, (1988).

\section{The Binary Star Gamma Persei — Bright, but Ill-Understood}

\author{
R.E.M. Griffin
}

\section{HIA/DAO, Victoria, Canada}

Gamma Per looks like an unexceptional composite-spectrum binary, most often classified as G8 III + A3 V. Nevertheless, early studies indicated that the masses of the component stars were on the high side, until Popper showed that the component stars were not overmassive so much as over-luminous. Pourbaix then went further, constraining the masses on the basis of evolutionary theory and largely removing any discrepancies in mass. However, recent CCD spectroscopy, from which the secondary spectrum has been fully isolated by a spectrum subtraction technique, finds that the masses of the individual components $\left(3.9 \mathrm{M}_{\odot}\right.$ for the giant and $2.5 \mathrm{M}_{\odot}$ for the dwarf) are indeed greater than would be expected from their spectral types, thus re-opening the question of the origin and evolution of this somewhat unusual system.

Analyses of more than 25 composite-spectrum systems by spectrum subtraction have now produced enough results that a picture is beginning to emerge - and it is an unexpected one: like $\gamma$ Per, many other systems also have secondary stars that have started evolving away from the main-sequence, thus challenging us to explain how the two stars can have attained that state of rapid evolution and yet be of the same age. The importance of the new analysis of gamma Per is the high precision with which the observed stellar parameters of one of these systems can be determined. 


\title{
The Population of Close Binaries Dynamically Formed in Hierarchical Triple Systems, with Application to Extrasolar Planets
}

\author{
Daniel C. Fabrycky \\ Princeton University, Princeton, NJ, United States
}

Because of the large radii of pre-main-sequence stars, the current separation of close binaries was not likely established at their formation. The secular perturbation of a third star orbiting a binary at high inclination can cause its eccentricity to grow close to unity as angular momentum is gained by the third star. Close pericenter passages at high eccentricity cause dissipative tides, and the loss of orbital energy can result in a close binary. A population of triple stars is considered, of which some will undergo this evolution. The distribution of binary period and of relative inclination of the third star's orbit to the binary plane is derived using a Monte-Carlo method. The averaged (secular) equations of motion are integrated, including a model for tidal dissipation and extra precession due to the tidal and spin distortion of the stars and general relativity. The predicted period distribution matches reasonably to a set of spectroscopic binaries with known companions: there is an excess of such binaries with periods below about 5 days relative to the population of true binary systems. Reasonable statistics for the relative inclinations of such triple stars will be built up by optical interferometers in the near future, and these observations can check the predicted distribution.

This mechanism is also applicable to extrasolar planets hosted by binary stars, causing them to migrate to short periods. For planets with periods less than 20 days, there is a paucity of massive (minimum mass $>2$ Jupiter mass) planets orbiting single stars, yet there are three such planets (Tau Boo b, HD 195019b, Gl 86b) in binaries. Therefore the single-star migration mechanism is apparently sensitive to planetary mass, whereas the binary-star migration mechanism is not; the latter matches the present study. Furthermore, HD 195019b and Gl 86b have circularized orbits, whereas single-star planets with comparable periods show a range of eccentricities; planetary radius inflation resulting from the epoch of high eccentricity may have led to this efficient circularization.

\section{The UV Spectrum of the Binary Star 88 Her: Activity Cycles in the Circumstellar Envelope}

\author{
A. Granada ${ }^{1}$, L. Cidale ${ }^{1,2}$, and C. Quiroga ${ }^{1,2}$ \\ ${ }^{1}$ Falcultad de Ciencias Astronómicas y Geofísicas, UNLP, Argentina, \\ ${ }^{2}$ Instituto de Astrofísica La Plata, CONICET
}

Since its discovery as a variable star, 88 Her has undergone three long-term photometric variation cycles with transitions between Be-shell and normal B phases. From the spectroscopic study of fifteen high resolution spectra obtained by the IUE satellite between 1981 and 1992 we were able to set parameters such as optical depths and location of line forming regions. We also found that the periodic radial velocity variations of UV Fe II lines agree with the binary orbital period of 86.7 days (Harmanec et al., 1974) and that the line absorption depth variations have a cycle of about 1560 days. Our aims are to relate the properties of the circumstellar envelope of 88 Her to the spectroscopic variability observed in Fe II and Mg II UV lines, and to understand the mechanisms which cause them, as well as the influence that binarity has on them. 